\title{
THE CONTRIBUTION OF GALACTIC FREE-FREE EMISSION TO ANISOTROPIES IN THE COSMIC MICROWAVE BACKGROUND FOUND BY THE SASKATOON EXPERIMENT
}

\author{
John H. Simonetti, Brian Dennison, and Gregory A. Topasna \\ Martin Observatory, Institute for Particle Physics and Astrophysics, and Department of Physics, Virginia Polytechnic Institute and State University, \\ Blacksburg, VA 24061 \\ Received 1995 October 24; accepted 1995 November 30
}

\begin{abstract}
We made a sensitive, wide-field $\mathrm{H} \alpha$ image of the north celestial polar region. Using this image, we constrain the contribution of irregularities in interstellar free-free emission to the degree-scale anisotropies in the cosmic microwave background detected in recent observations at Saskatoon by the Princeton group. The analysis of the $\mathrm{H} \alpha$ image mimics the Saskatoon data analysis: the resulting signal is the strength of irregularities sampled with the Saskatoon beam (i.e., degree-scale) along the $85^{\circ}$ declination circle. We found no such irregularities that could be attributed to $\mathrm{H} \alpha$ emission. The implied upper bound on the rms variation in free-free brightness temperature is less than $4.6 \mu \mathrm{K}$ at $27.5 \mathrm{GHz}$. The observed cosmic microwave background anisotropies are much larger. Therefore, the contribution of irregularities in interstellar free-free emission to the observed anisotropies is negligible.
\end{abstract}

Subject headings: cosmic microwave background — instrumentation: miscellaneous — ISM: general

\section{INTRODUCTION}

Anisotropies in the cosmic microwave background radiation $(\mathrm{CMB})$ on scales greater than $7^{\circ}$ were discovered in the COBE DMR experiment (Smoot et al. 1992; Bennett et al. 1992). Ground-based searches for anisotropies in the CMB on degree and smaller scales are currently underway. One such search, made from Saskatoon, SK, Canada, has yielded positive results (Wollack et al. 1993; Netterfield et al. 1995). Although the contribution to those irregularities from foreground Galactic free-free emission can be constrained by multifrequency observations, it is important to use observations of the interstellar Balmer-alpha $(\mathrm{H} \alpha)$ emission to infer the extent of contamination. Here we report on $\mathrm{H} \alpha$ observations toward the celestial polar cap which we use to constrain the free-free contribution to the results of the Saskatoon experiment. The analysis of our $\mathrm{H} \alpha$ images closely mimics the microwave observations and analysis performed by the Princeton group, yielding information intimately related to their results. The contribution from irregularities in the interstellar free-free emission is very small in comparison with the observed microwave anisotropies. Gaustad et al. (1995) have taken several $\mathrm{H} \alpha$ images of the same region; they report an upper limit to the rms in their images equivalent to $<4 \mu \mathrm{K}$ at $30 \mathrm{GHz}$, which is consistent with our analysis.

\section{OBSERVATIONS}

Using a wide-field camera and narrow-bandpass interference filters, we imaged the $\mathrm{H} \alpha$ emission in the north celestial polar region. Details of the instrumentation will be described elsewhere. The basic instrument consists of a cryogenically cooled, TK $512 \times 512$ CCD with $27 \mu \mathrm{m}$ pixels placed in the focal plane of a fast (f/1.2) photographic lens. A filter wheel placed ahead of the lens enables imaging with both a narrow-bandpass $\mathrm{H} \alpha$ filter $(6566 \AA$ center wavelength and $17.5 \AA$ bandpass) and a wider bandpass continuum filter (6083 Å center wavelength and $80 \AA$ bandpass) in a line-free part of the spectrum. For very wide angle images, such as those discussed in this Letter, an additional fieldenlarging lens system is mounted ahead of the filter wheel. The resulting images have a usable angular diameter of about $30^{\circ}$, with pixel sizes of about 6:7. The effective passband for narrowband interference filters shifts downward in wavelength for off-axis rays, but the center wavelength and bandpass of the $\mathrm{H} \alpha$ filter allows the imaging of $\mathrm{H} \alpha$ emission with a line-of-sight velocity within (at least) $\pm 100 \mathrm{~km} \mathrm{~s}^{-1}$ anywhere within the $30^{\circ}$ field. The system can detect $\approx 1$ rayleigh (at $5 \sigma$ ) within a single pixel for a $2 \mathrm{hr}$ integration ( 1 rayleigh $=1 \mathrm{R}=10^{6} / 4 \pi$ photons $\mathrm{cm}^{-2} \mathrm{~s}^{-1}$ $\left.\mathrm{sr}^{-1}\right)$.

The imaging system is equatorially mounted; tracking to arcsecond precision (much better than necessary) is assured through the use of a separate auto-tracking CCD. The system is mounted on an enclosed concrete pier at the Martin Observatory on the grounds of the Miles C. Horton, Sr., Research Center, located in an isolated region of southwestern Virginia.

On the night of 1995 April 26/27 we obtained twelve 10 minute $\mathrm{H} \alpha$ images and two 10 minute continuum images of the north celestial pole (NCP). We also took one 10 minute $\mathrm{H} \alpha$ image centered on $\alpha \approx 20^{\mathrm{h}} 10^{\mathrm{m}}, \delta \approx+32^{\circ}$ (the CygnusLyra region) for calibration purposes. We recorded a set of 10 flat-field images with each filter. Given the wide-field nature of the images, we did not use sky flats. Instead, we made the flat-field images by pointing the system at a wide "light box" containing a smooth distribution of small incandescent bulbs and covered with two separated layers of translucent glass; a third layer of translucent glass was placed in front of the field-enlarging lens system. Previous experimentation with the number and spacing of the translucent sheets yielded consistent flat fields with negligible sensitivity to the actual arrangement of the components. In addition to the flat fields, we recorded 10 zero-integration bias images. Due to the very low dark current of the system (about 0.001 electrons s ${ }^{-1}$ pixel $^{-1}$ ), "dark" images were not necessary. 


\section{IMAGE REDUCTION}

For the initial processing of the images we used IRAF. ${ }^{1} \mathrm{We}$ averaged the 10 recorded bias images, ignoring the highest pixel value at each specific pixel location, thus removing pixel defects caused by cosmic-ray hits. In the same way, we averaged 10 bias-corrected flat-field images for each filter. The resulting final flat-field images and bias image were used to correct the individual 10 minute sky integrations. We produced a final $\mathrm{H} \alpha$ image and continuum image by averaging the fully corrected 10 minute integrations, again throwing out the highest pixel value at each pixel location. The final $\mathrm{H} \alpha$ image of the NCP was effectively the result, therefore, of a 110 minute integration (Fig. 1 [Pl. L1]).

To convert the pixel values of the $\mathrm{NCP} \mathrm{H} \alpha$ image into rayleighs, we used the $\mathrm{H} \alpha$ flux of the planetary nebula IC 3568 , which is near the NCP. We checked the calibration using the planetary nebula NGC 6720 (M57) in the $\mathrm{H} \alpha$ image of the Cygnus-Lyra region and observed at about the same altitude as the NCP. We had previously tested this scheme of using planetary nebulae for calibration by comparing calibrations derived from both the planetary nebula NGC 7027 and the North America Nebula (lying in the same image); the results agreed to within $10 \%$. An $\mathrm{H} \alpha$ intensity for the North America Nebula is given by Scherb (1981). $\mathrm{H} \alpha$ fluxes of the planetary nebula were determined from Cahn, Kaler, \& Stanghellini (1992). Calibration using planetary nebulae or the North America Nebula lead to consistent results.

We determined a transformation between pixel coordinates $(x, y)$ and right ascension and declination in the $\mathrm{H} \alpha$ image of the NCP by using the known positions of a set of bright stars.

To reduce the effect of stars on the search for irregularities in the $\mathrm{H} \alpha$ emission toward the NCP, we subtracted a scaled version of the red continuum image from the $\mathrm{H} \alpha$ image. Without subtracting stars, an attempt to quantify the irregularities in our $\mathrm{H} \alpha$ image by, for example, taking a simple rms would lead to a misleadingly large result. The scaling was necessary to account for the difference in passband width of the two filters, and the difference in flux per unit wavelength at the central wavelengths of the two filters for the bright stars that would most affect the results. Based on the peak pixel values in the $\mathrm{H} \alpha$ and continuum images for a set of representative stars, we divided the continuum image by a factor of 5 before subtraction from the $\mathrm{H} \alpha$ image. The resulting difference image is nearly devoid of stellar images. The difference image was used in all subsequent analysis; we will refer to it hereafter simply as the $\mathrm{H} \alpha$ image. This scaling and differencing scheme does not totally remove every star's image-using a single scaling number does not take account of star-to-star variations in spectral type and, therefore, flux density at the two wavelengths. As we shall see, much of the signal in our final result is due to stellar residuals: our results represent an upper limit to the $\mathrm{H} \alpha$ anisotropies. However, the limit we obtained is so stringent, we decided it was unnecessary to experiment further with star removal at this time.

\section{ANALYSIS}

The subsequent analysis of the $\mathrm{H} \alpha$ image mimicked the analysis performed in the Saskatoon experiments (Wollack et al. 1993). To give the $\mathrm{H} \alpha$ image the same resolution as the

${ }^{1}$ IRAF is distributed by the National Optical Astronomy Observatories, which is operated by the Association of Universities for Research in Astronomy, Inc., under cooperative agreement with the National Science Foundation.
Saskatoon radio telescope, we convolved the $\mathrm{H} \alpha$ image with a 1.45 FWHM Gaussian beam, using IRAF (Fig. 2 [P1. L2]). In the Saskatoon experiment, the radio beam was chopped in azimuth at $3.91 \mathrm{~Hz}$ and the data stream was weighted so as to produce a triple beam with one positive lobe and two negative lobes on either side, and zero total power. To simulate one chop cycle, we sampled our smoothed $\mathrm{H} \alpha$ map at a series of pixel coordinates corresponding to one sinusoidal oscillation in azimuth at a fixed altitude $\left(52^{\circ} .15\right.$ - the latitude of Saskatoon), with appropriate weighting of the samples.

As an approximation to the continuous chopping motion of the radio telescope, we sampled the smoothed $\mathrm{H} \alpha$ image throughout 240 single-cycle chops which were equally spaced in starting right ascension between $0^{\mathrm{h}}$ and $24^{\mathrm{h}}$. The center azimuth for the radio beam's chopping motion was 8.0 from north; the amplitude of the chopping was 4.0 (in azimuth). ${ }^{2}$ Therefore, the starting declination for sampling the $\mathrm{H} \alpha$ image during a single chop was $87^{\circ} .5$, and halfway through the chop the lowest declination was reached at $82^{\circ} .6$. During one single-cycle chop, the image was sampled at 128 locations equally spaced in phase throughout the period of the chop. We determined the $\mathrm{H} \alpha$ value for a sample by interpolating from the pixels surrounding the sample location.

The 128 samples in a single-cycle chop were binned in groups of eight; the eight samples in each bin were averaged, yielding 16 data points for the chop. Finally, one output signal value for a single-cycle chop was obtained by multiplying the set of 16 data points by a corresponding set of 16 weights, and summing. The weights produce a demodulation of the data and are given by a normalized sinusoidal function with twice the rate of the chop. In effect, the output signal value is equivalent to the output of the "double difference" beam of the Saskatoon analysis (a positive beam at about $85^{\circ}$ declination flanked by two negative beams of half the amplitude and lying at declinations of about $82^{\circ}$ and $87^{\circ}$ ). This output signal is relatively insensitive to smooth backgrounds (e.g., uniform or gradients). The normalization ensures that a $1 \mathrm{R}$ source filling the positive beam in an otherwise smooth sky will yield a $1 \mathrm{R}$ signal.

We binned the 240 output signal values by right ascension and averaged within the bins to produce one result for each hour of right ascension. (Performing the full analysis using only 1 chop per right ascension bin yielded essentially the same results, which is not surprising given the 1.45 convolution of the original image.) Before plotting, we subtracted the mean signal $(-0.36 \mathrm{R})$ across the 24 right ascension bins. Figure 3 displays a plot of the signal as a function of right ascension bin. The rms variation in the plotted signal is $0.58 \mathrm{R}$. These irregularities are readily traced to incompletely subtracted stars. Thus, they imply an upper limit to the fluctuations in $\mathrm{H} \alpha$ emission and the corresponding free-free emission sampled by the Saskatoon beam.

\section{DISCUSSION}

To report their observations of anisotropies in the CMB made from Saskatoon, Wollack et al. (1993) and Netterfield et al. (1995) use plots similar to Figure 3. For direct comparison

2 The radio beam was chopped at center locations both $8: 0$ east and $8: 0$ west of north. The east and west results for a given right ascension were averaged. For simplicity, our analysis uses only the results of the western chopping location. 


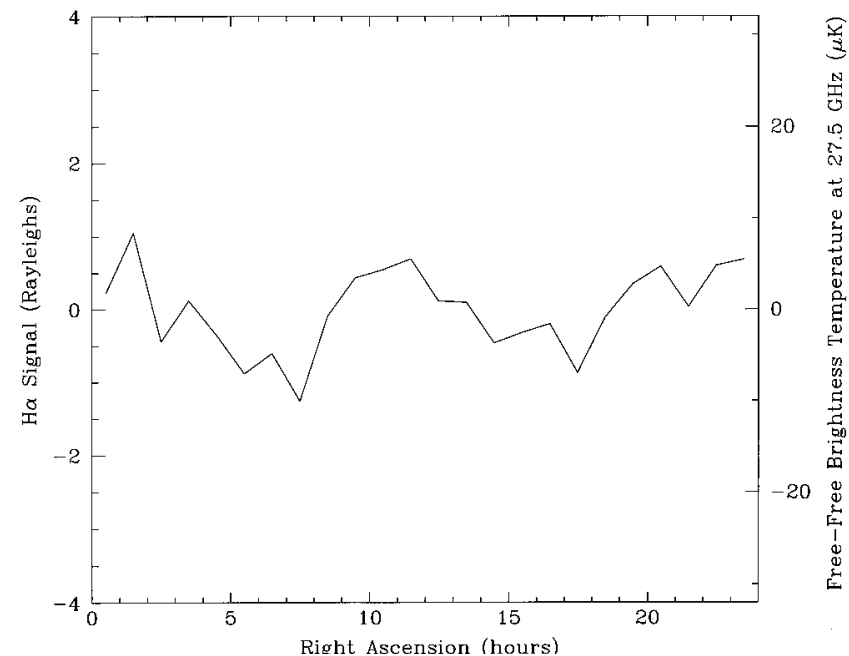

FIG. 3.-Output signal (in rayleighs) resulting from sampling the $\mathrm{H} \alpha$ image of Fig. 2 throughout a chopping pattern similar to that used in the Saskatoon experiment (see $\S 4$ ). Also shown is the implied Galactic free-free brightness temperature scale at $27.5 \mathrm{GHz}$ (see \$5). The signal is the strength of degree-scale irregularities in the image along a full right ascension circle at a declination of about $85^{\circ}$. Compare to Fig. 1 from Wollack et al. (1993) and Fig. 2 from Netterfield et al. (1995).

with our results, we have added a scale to Figure 3 for the implied free-free brightness temperature at $27.5 \mathrm{GHz}$.

The free-free brightness temperature (in $\mu \mathrm{K}$ ) of optically thin, ionized hydrogen gas is

$$
T_{b}(\mu \mathrm{K})=\frac{5.43}{\nu_{10}^{2} T_{4}^{1 / 2}} g_{\mathrm{ff}} E,
$$

where $\nu_{10}$ is the frequency in units of $10 \mathrm{GHz}, T_{4}$ is the gas temperature in units of $10^{4} \mathrm{~K}, E$ is the emission measure in units of $\mathrm{pc} \mathrm{cm}{ }^{-6}$, and $g_{\mathrm{ff}}$ is the free-free Gaunt factor (Osterbrock 1989). The same gas produces an $\mathrm{H} \alpha$ surface brightness (in rayleighs) of

$$
I(\mathrm{R})=0.36 E T_{4}^{-1 / 2}\left(1-0.37 \ln T_{4}\right)
$$

(Bennett et al. 1992), assuming no extinction. Therefore, we have

$$
T_{b}(\mu \mathrm{K})=14.9 \frac{g_{\mathrm{ff}} I(\mathrm{R})}{\nu_{10}^{2}\left(1-0.37 \ln T_{4}\right)} .
$$

The brightness temperature $T_{b}$ is not strongly dependent upon the gas temperature; we assume $\mathrm{T}_{4}=1$ in what follows.
As stated above, equation (3) assumes any observed $\mathrm{H} \alpha$ emission is unaffected by interstellar extinction. If extinction is significant, the microwave brightness temperature is higher than given by equation (3). The $100 \mu \mathrm{m}$ intensities observed by IRAS within $10^{\circ}$ of the north celestial pole are $\lesssim 10 \mathrm{MJy} \mathrm{sr}^{-1}$ (Wheelock et al. 1994), implying $\lesssim 0.6$ mag of visual extinction (Beichman 1987). Therefore, for this field, the brightness temperature calculated by equation (3) should be increased by $60 \%$, at most. The full $60 \%$ adjustment would apply only if the dust detected by IRAS lies entirely in front of any $\mathrm{H} \alpha$-emitting gas. A less than $60 \%$ increase in brightness temperature does not affect our final conclusions (see below). We have used equation (3) to set the brightness temperature scale in Figure 3 , and in calculating our quantitative results.

The Saskatoon observations were made at $K_{a}$ and $Q$ bands. A frequency of $27.5 \mathrm{GHz}$ is representative of the $K_{a}$-band observations (i.e., the lower frequency band) and is used to set the brightness temperature scale on the right-hand side of Figure 3. Using this scale, the rms variation in the plotted values is $4.6 \mu \mathrm{K}$. In comparison, rms variations of $\approx 40 \mu \mathrm{K}$ are present in the $K_{a}$ - and $Q$-band results reported by Wollack et al. (1993) and Netterfield et al. (1995). If we use $44.5 \mathrm{GHz}(Q$ band), the rms of our signal becomes $1.7 \mu \mathrm{K}$. Figure 2 in Netterfield et al. (1995) displays, in one plot, the results of both the $K_{a}$ - and $Q$-band observations, and can be easily compared with our Figure 3. At either band, our results are an upper limit to the actual free-free emission irregularities.

Based on our $\mathrm{H} \alpha$ observations and this analysis, irregularities in the Galactic free-free emission toward the north celestial pole make a negligible contribution to the CMB anisotropies observed in the Saskatoon experiment.

The authors thank David Dupuy for assistance with initial equipment tests, and David Wilkinson and Lyman Page for valuable discussions and for providing algorithms for simulating the Saskatoon beam. The authors especially thank Mr. and Mrs. Miles C. Horton, Jr., for their encouragement and enthusiastic support of this research. This work was supported by National Science Foundation grant AST-9319670, and a grant from the Horton Foundation to Virginia Polytechnic Institute and State University. The Miles C. Horton, Sr., Research Center, located near Mountain Lake, Virginia, is operated by Virginia Polytechnic Institute and State University with support from the Horton Foundation. Technical assistance with the imaging system was provided by ElectroTec Corporation.

\section{REFERENCES}

Bennett, C. L., et al. 1992, ApJ, 396, L7

Beichman, C. A. 1987, ARA\&A, 25, 521

Cahn, H. H., Kaler, J. B., \& Stanghellini, L. 1992, A\&AS, 94, 399

Gaustad, J. E., Oh, E. S., McCullough, P. R., \& Van Buren, D. 1995, BAAS, 27, 823

Netterfield, C. B., Jarosik, N., Page, L., Wilkinson, D., \& Wollack, E. 1995, ApJ, 445, L69

Osterbrock, D. E. 1989, Astrophysics of Gaseous Nebulae and Active Galactic Nuclei (Mill Valley: University Science Books)
Scherb, F. 1981, ApJ, 243, 644

Smoot, G. F., et al. 1992, ApJ, 396, L1

Wheelock, S., et al. 1994, IRAS Sky Survey Atlas Explanatory Supplement (JPL Publ. 94-11; Pasadena: JPL)

Wollack, E. J., Jarosik, N. C., Netterfield, C. B., Page, L. A., \& Wilkinson, D 1993, ApJ, 419, L49 



\section{PLATE L1}

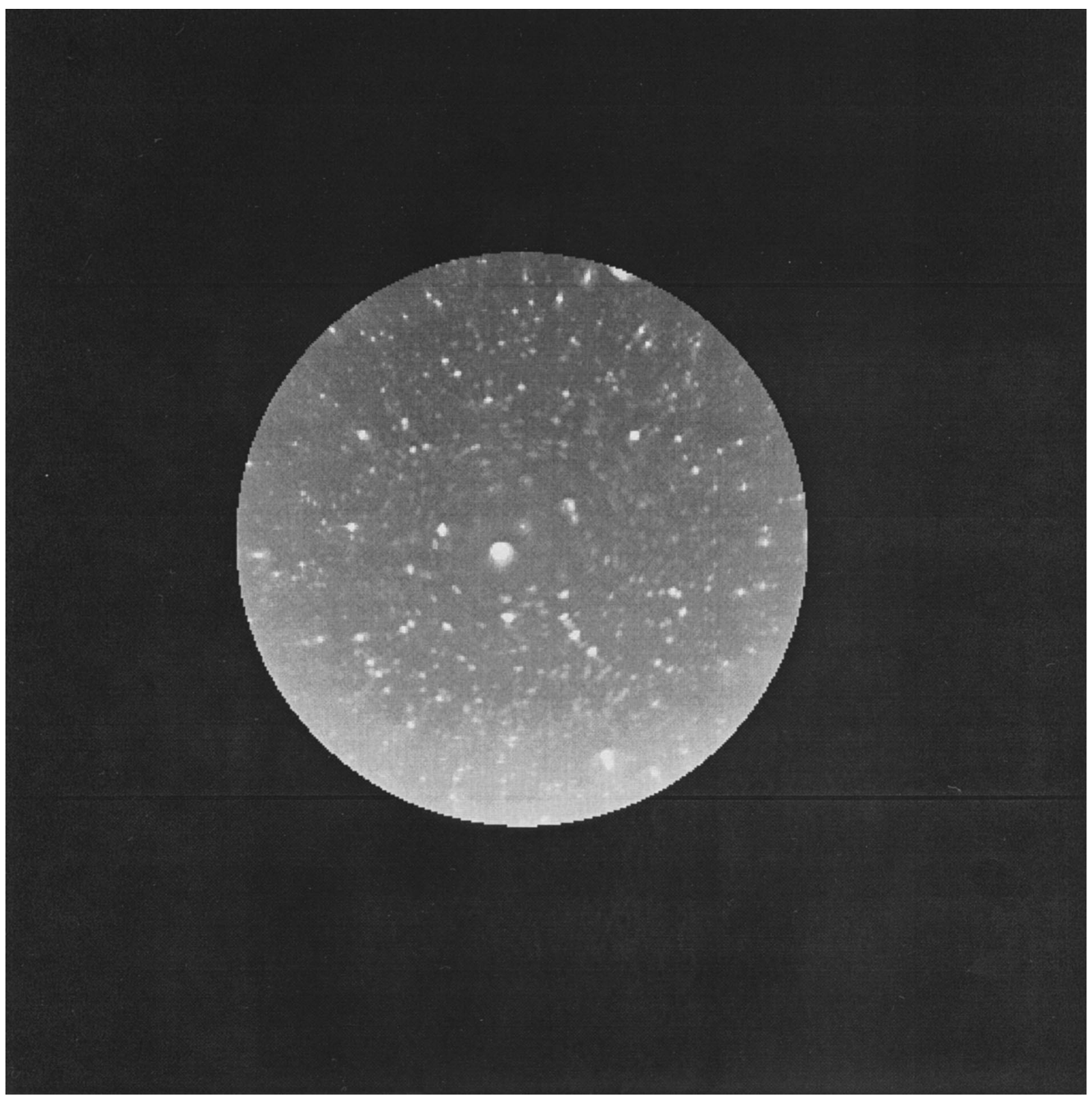

FIG. 1.-Image of the NCP region taken with the $\mathrm{H} \alpha$ filter (equivalent to a 110 minute integration). The diameter of the field is $30^{\circ}$. Polaris is the bright star near the field center; the $1^{\mathrm{h}}$ right ascension line runs approximately vertically from the center to the bottom edge of the image and is nearly parallel to a line of constant Galactic longitude. The bottom edge of the image is $\approx 12^{\circ}$ from the Galactic plane.

Simonetti, Dennison, \& Topsana (see 458, L2) 
PLATE L2

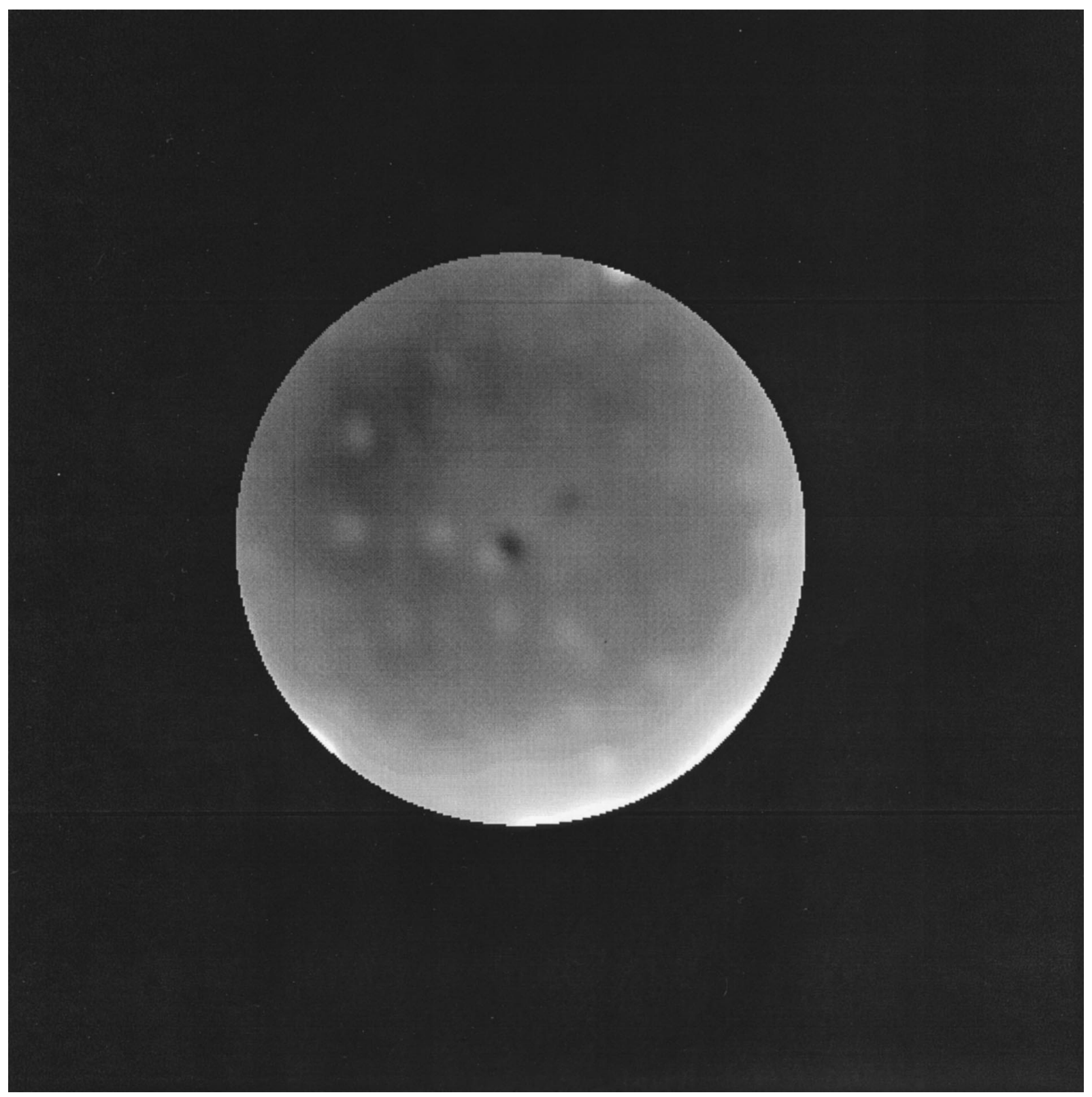

FIG. 2.- $\mathrm{H} \alpha$ image of the NCP region after subtraction of the scaled continuum image, and smoothing with a 1:45 FWHM beam. Most visible irregularities are traceable to incompletely subtracted stars. The minimum pixel value displayed is about $10 \%$ of the minimum value displayed in Fig. 1, and the range of displayed pixel values is $25 \%$ of that in Fig. 1. Incomplete subtraction of Polaris is responsible for the local minimum near the image center (and the adjacent local maximum). The bright spot directly below Polaris (at $\delta \approx 86^{\circ}, \alpha \approx 1^{\mathrm{h}}$ ) is due to incomplete subtraction of the star $2 \mathrm{UMi}$; the spot has a brightness of about $1.5-2 \mathrm{R}$ above the local background and is responsible for the maximum in the signal of Fig. 3 at right ascension $\approx 1^{\mathrm{h}}$. The increased brightness along the bottom edge of the image is due to light from the Galactic plane.

Simonetti, Dennison, \& Topsana (see 458, L2) 